See discussions, stats, and author profiles for this publication at: https://www.researchgate.net/publication/330197775

\title{
Single-Stage Drag Modulation GNC Performance for Venus Aerocapture Demonstration
}

Conference Paper · January 2019

DOI: 10.2514/6.2019-0016

CITATIONS

READS

5

32

3 authors, including:

Evan Roelke

University of Colorado Boulder

9 PUBLICATIONS 18 CITATIONS

SEE PROFILE

Some of the authors of this publication are also working on these related projects:

Project Multi-Event Drag Modulated Aerocapture Guidance View project 


\title{
Single-Stage Drag Modulation GNC Performance for Venus Aerocapture Demonstration
}

\author{
Roelke, E.* Braun, R.D. ${ }^{\dagger}$, and Werner, M.S. ${ }^{\ddagger}$ \\ University of Colorado Boulder
}

\begin{abstract}
The future of space exploration is heavily reliant on innovative Entry, Descent, and Landing (EDL) technologies. One such innovation involves aeroassist technology, mainly aerocapture, which involves a single pass through the upper atmosphere of a planetary body from an incoming hyperbolic trajectory to capture into an elliptical orbit. Previous aerocapture guidance studies focused on lift modulation using reaction-control system (RCS) thrusters. This paper investigates the guidance law performance of drag-modulated aerocapture for a single, discrete, jettison event. Venus is selected as the target planet to observe performance in the most challenging environment. Three different guidance algorithms are studied, including a deceleration curve-fit, a pure state-predictor, and a numerical predictor-corrector utilizing both bisection and Newton-Raphson root-finding methods. The performance is assessed using Monte Carlo simulation methods. The deceleration curve-fit achieves a capture rate of over $55 \%$ for a $2000 \mathrm{~km}$ apoapsis target and $1000 \mathrm{~km}$ tolerance. For an entry flight-path-angle (EFPA) of $\mathbf{- 5 . 2}{ }^{\circ}$, the predictive algorithm achieves a 50\% capture rate within $100 \mathrm{~km}$ and over $\mathbf{9 0 \%}$ within $250 \mathrm{~km}$. Finally, at the same EFPA the NPC using a Newton method attains $>\mathbf{8 0} \%$ capture rate for all $\beta_{2} / \beta_{1}>6$ within $100 \mathrm{~km}$ tolerance targeting $10000 \mathrm{~km}$. The largest source of error for these systems is the atmospheric density variation downstream of the jettison event.
\end{abstract}

\section{Nomenclature}

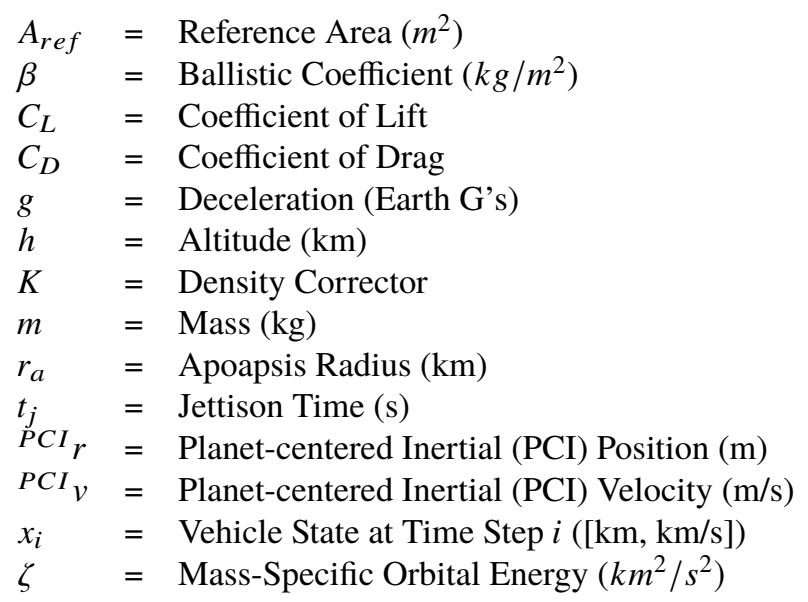

\section{Introduction}

EROASSIST maneuvers provide a promising innovation to Entry, Descent, and Landing
the upper atmosphere of a planetary body to decrease the spacecraft’s orbital energy with
The two main cases of aeroassist maneuvers for orbital operations are aerobraking and aeroca
below; the former involves multiple passes through the upper atmosphere of a planetary

${ }^{*}$ Graduate Research Assistant, Entry systems Design Lab, Colorado Center for Astrodynamics Research.
${ }^{\dagger}$ Smead Professor of Space Technology, Dean of Engineering and Applied Science
${ }^{\ddagger}$ Graduate Research Assistant, Entry systems Design Lab, Colorado Center for Astrodynamics Research. 
lower the spacecraft apoapsis until a desired science orbit is achieved. Aerobraking is limited by payload mass, requires an initial orbital insertion maneuver, and increases the duration of time until a nominal science orbit is attained. The latter maneuver type, aerocapture, depicted in Figure $1 \mathrm{~b}$, is initiated from an inbound hyperbolic trajectory and only requires a single pass through the upper atmosphere to capture into a nominal orbit about the central body, thereby saving time and increasing payload mass and/or reducing propellant mass.

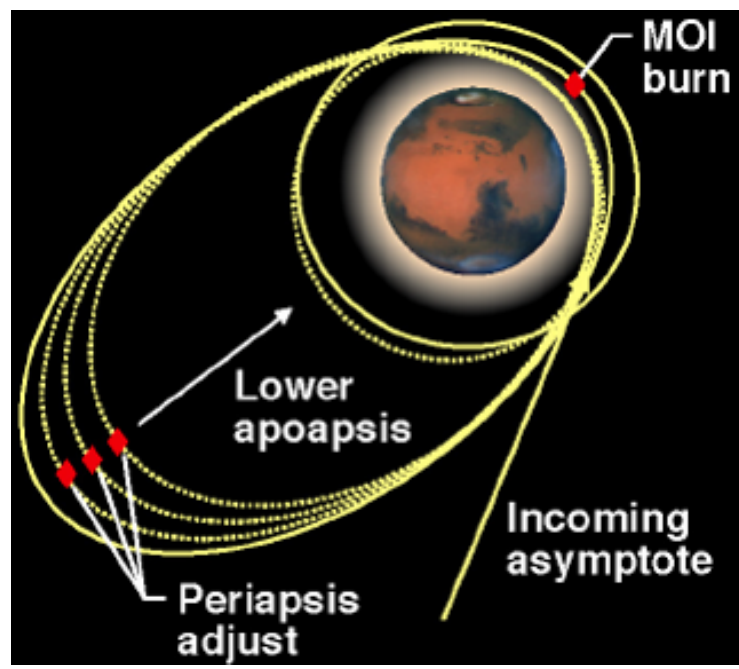

(a) Aerobraking Maneuver Diagram

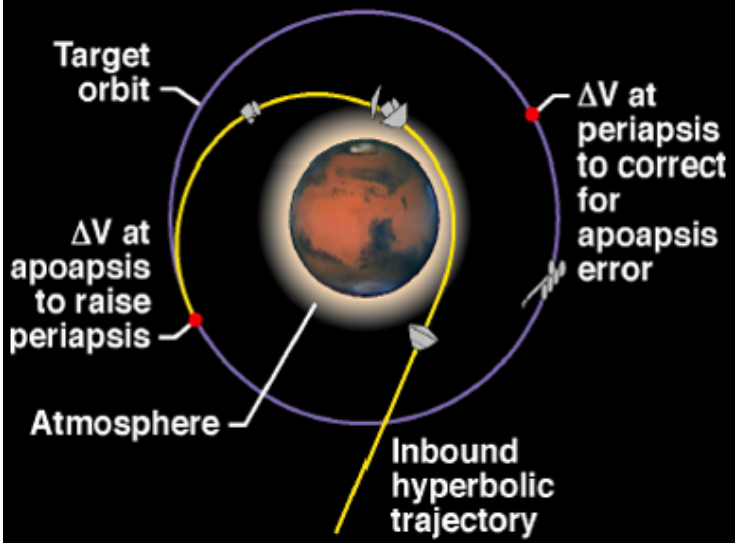

(b) Aerocapture Maneuver Diagram

Fig. 1 Aeroassist Maneuver Diagrams [1]

This maneuver will then require a periapsis raise maneuver upon reaching the resultant orbit apoapsis, as the closest approach of the current orbit still lies inside the atmosphere. Apoapsis error adjustment operations can then be performed at periapsis, as shown in Figure $1 \mathrm{~b}$.

Generally speaking, aerocapture has been shown to provide significant mass savings at all planetary bodies, even enabling a certain set of missions to the outer gas and ice giants. More specifically, aerocapture has been shown to increase deliverable mass by over $40 \%$ to an elliptical orbit at Venus based on Magellan, and over $70 \%$ for a low-altitude circular orbit at Venus [2].

\section{A. Discrete-Event Drag-Modulation Aerocapture}

While aerobraking enables the system to implement multiple control events over multiple atmospheric passes, the control authority during aerocapture must be performed during the single atmospheric pass in order to target a specific orbit. Guidance laws for this atmospheric pass can be categorized by lift-modulation or drag-modulation, with each case being split into either discrete-event or continuously-variable control. Aerocapture studies in the past have thoroughly analyzed lift-modulation aerocapture, utilizing reaction control system (RCS) thrusters to rotate the vertical lift vector out-of-plane for range control. However, this requires additional mass to offset the vehicle center of mass in order to fly at an angle of attack, as well as a complex guidance and control laws to steer the vehicle through the atmosphere [3] [4] [5] [6].

Drag-modulation aerocapture is promising because the vehicle can enter the atmosphere in a ballistic configuration (zero angle-of-attack), and the control inputs directly and promptly affect the vehicle's ballistic coefficient, $\beta=\frac{m}{C_{D} A_{r e f}}$. This parameter can be interpreted as a ratio of inertial forces to aerodynamics forces; in other words, how predominantly the vehicle responds to changes in aerodynamics forces. A small $\beta$ refers to a system that feels large drag forces and will slow down much quicker in the atmosphere, whereas a large $\beta$ will take much longer to be impeded by such aerodynamic loads.

Discrete-event drag modulation, depicted in Figure 2 is the simplest of the aforementioned types of control for aerocapture due to the simplicity of the vehicle and the associated guidance and control laws. During this maneuver, a drag skirt is separated from the main body at a specific instant during the trajectory, altering the vehicle's $\beta$, thus changing the amount of orbital energy, $\zeta$, lost during its atmospheric flight. The amount of energy lost during the atmospheric pass is dependent on the timing of the jettison event, enabling the system to target a specific orbit. Because 


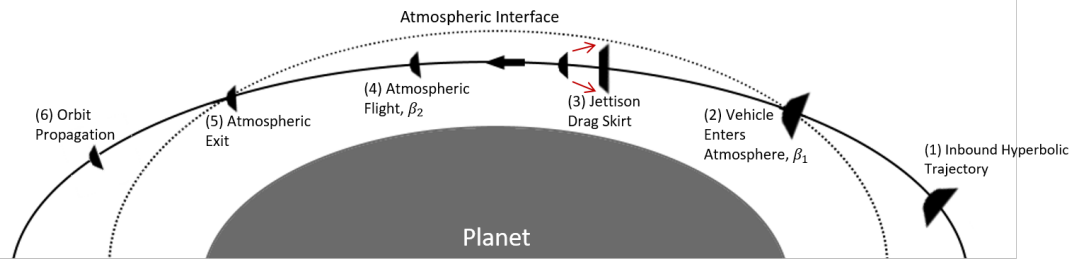

Fig. 2 Single-Stage, Discrete-Event, Drag-Modulation Aerocapture Maneuver Diagram

the jettison event will reduce the vehicle's $\beta$, if the jettison event occurs too early in the trajectory, the resultant apoapsis will rest above the desired altitude, possibly continuing along a hyperbolic orbit. Similarly, if the jettison event occurs too late in the trajectory, the vehicle will have lost too much energy and the resultant apoapsis will fall below the desired altitude, and may even impact the surface.

While single-stage drag-modulation systems are relatively simple architectures, their performance is limited by lack of control authority; once the jettison event has occurred, the vehicle will have exhausted all control options and will be vulnerable to perturbations from atmospheric or vehicle uncertainties. A potential solution to this problem involves multiple jettison events, as discussed in Section $\mathrm{V}$

\section{B. Aerocapture at Venus}

As far as targets for aerocapture are concerned, Venus should be considered an "edge case." The high atmospheric temperature heavily constrains the thermal protection systems (TPS). In addition, the high atmospheric density contributes to large apoapsis errors for small jettison time differences during the trajectory, particularly for single-stage systems that lose all further control authority once they have jettisoned the drag skirt. The large uncertainty in the atmospheric properties thus require a highly accurate, computationally inexpensive guidance system that can appropriately respond to perturbations from the nominal trajectory before the jettison event.

The justification for choosing such a difficult scenario with which to study drag-modulated aerocapture stems from its complexity; if aerocapture can be proven feasible at this planetary body, then logically it should be feasible at all other potential targets in the solar system. This investigation seeks to quantify the performance of various guidance algorithms in an effort to determine the efficacy of single-stage drag-modulation aerocapture for all planetary bodies by focusing on Venus.

\section{Methodology}

\section{A. Numerical Integration}

In order to quantitatively assess the performance of various guidance algorithms at Venus, a fourth-order Runge-Kutta integrator is written in Matlab to numerically integrate the system dynamics from atmospheric entry to exit. The Matlab code is then compiled into $\mathrm{C}++$ using the mex compiler for computational efficiency. The vehicle's planet-centered inertial position and velocity, ${ }^{P C I} r,{ }^{P C I} v$, at atmospheric exit can be converted to Keplerian orbital elements to determine the expected apoapsis radius. This way, the numeric integration is limited to atmospheric flight to reduce computation time.

The simulation suite models planetary bodies as oblate spheroids, using inverse-square and $\mathrm{J} 2$ perturbations to model the gravitational forces on the spacecraft. Venus Global Reference Atmospheric Model (GRAM) software was used to generate both nominal and disperse atmospheric property data tables as functions of altitude as detailed in Section II.B. The simulation uses linear interpolation to obtain atmospheric properties at each time step given the altitude of the spacecraft.

\section{B. Atmospheric Profiles}

VenusGRAM was used to generate a set of disperse atmospheric profiles, which were converted into tables for linear interpolation at each time step of the simulation. Figure 3 shows the atmospheric properties as functions of altitude along with the $\pm 3 \sigma$ boundary values. The large density variations shown in Figure 3 a will likely be a major source of apoapsis altitude error in the results detailed in Section[III. 


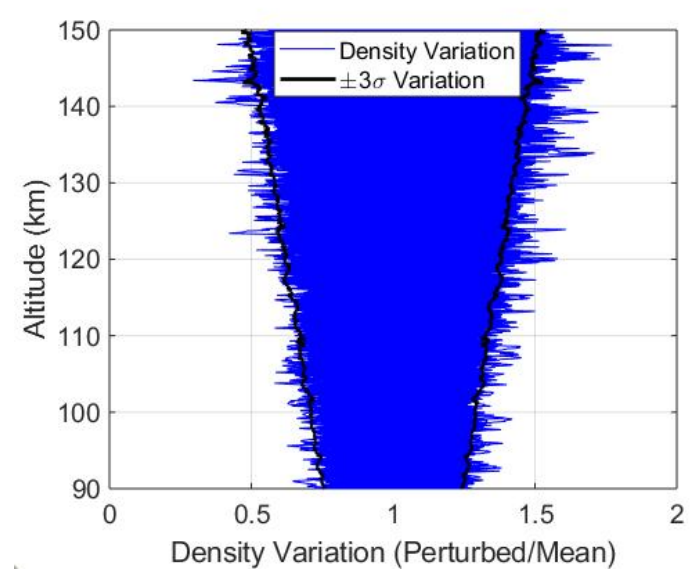

(a) Density Variations as Function of Altitude

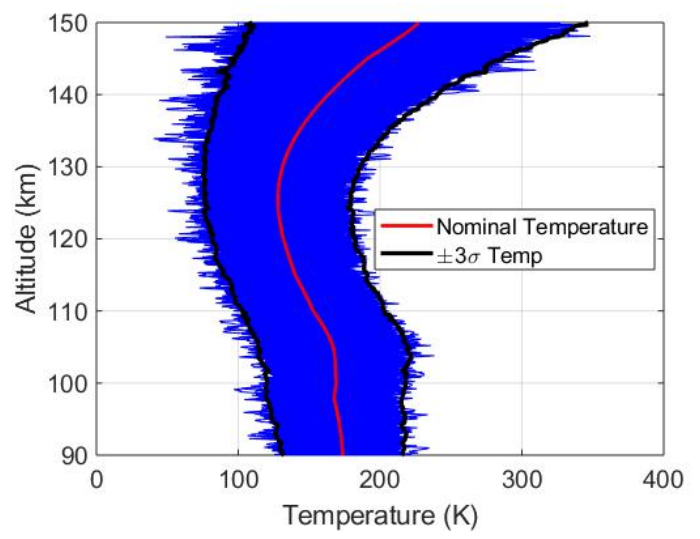

(b) Temperature Variations as Function of Altitude

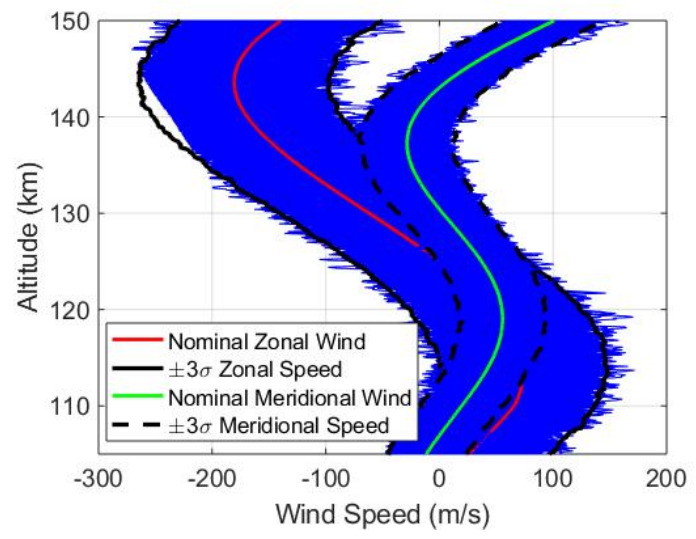

(c) Wind Speed Variations as Function of Altitude

\section{Fig. 3 Atmospheric Properties as Function of Altitude}

The uncertainty in temperature as shown in Figure 3b, while not necessarily relevant for a drag skirt jettison guidance algorithm, highlights the constraint on the TPS for performing aerocapture at Venus. Finally, Figure $3 \mathrm{c}$ shows $3 \sigma$ uncertainty in zonal wind speeds of over $100 \mathrm{~m} / \mathrm{s}$. While concerning for decelerator-equipped systems intending to land on planetary surfaces, wind speed tends to play a minimal role in aerocapture performance, as the vehicle is traveling primarily at hypersonic speeds.

\section{Guidance Algorithm Development}

Using the 4th-order Runge-Kutta integrator simulation suite, three different guidance algorithms are assessed in terms of their robustness and probability of capturing into orbit about Venus for a given apoapsis altitude tolerance. The first algorithm is a deceleration curve-fit (DCF) algorithm originally developed by Braun et. al. and adapted to aerocapture by Werner and Braun [7] [8]. The second is a pure-predictive guidance algorithm (PDG) derived from Miller et. al. for ballute-deployed aerocapture at Titan [9]. The third algorithm being assessed is a numerical predictor-corrector (NPC) algorithm originally developed for aerocapture by Putnam and Braun [10].

The guidance algorithms which use a predictor integration method, mainly the numerical predictor-corrector and pure-predictive algorithms, contain their own planetary models and atmospheric data tables rather than the main simulation parameters. This is done to simulate a real flight computer which will likely only have pre-loaded information on the nominal atmospheric properties. To accommodate for day-of-flight uncertainties, a density corrector term is implemented, described by Putnam and Braun, to allow the guidance system to adjust its estimated jettison time appropriately [10]. 


$$
K_{i+1}=K_{\text {gain }} K_{i}+\left(1-K_{\text {gain }}\right) K_{i}
$$

The density corrector is adjusted at each guidance time step, $i$, by the previous corrector value and a gain value, shown in equation 1 The density corrector is bounded by an upper and lower value to prevent extreme values if the gradient in the atmospheric density is too large.

\section{Deceleration Curve-Fit Algorithm}

The deceleration curve-fit algorithm, initially developed for Mars Pathfinder by Braun et. al., and applied to aerocapture by Werner and Braun, attempts to circumvent the need for a state-propagation predictor by using pre-loaded curve-fits to estimate a jettison time [7] [8].

The algorithm uses a deceleration curve fit as a function of entry flight-path angle (EFPA) to determine the optimal jettison time. An initial deceleration measurement value, $g_{1}$ and a $\Delta t$ value are used to find the time of the second deceleration measurement, $t_{g_{2}}=t_{g_{1}}+\Delta t$, that yields an optimal jettison time for a given EFPA. The optimal jettison time for each EFPA can be determined either by brute force or extremely high-fidelity NPC simulations; in this case the latter method was used, yielding jettison times for apoapsis errors below $1 \mathrm{~km}$ in each case. The unbiased nominal performance of the guidance algorithms is covered in more detail in Section II.C.4.

Using the deceleration values, $g_{1}$ and $g_{2}$, in addition to the time between measurements, a deceleration curve-fit is generated for a specific ballistic coefficient ratio, $\beta_{2} / \beta_{1}$, to correlate time until jettison, $t_{g o}$, with the value of $g_{2}$. The results for a $\beta_{2} / \beta_{1}=9.18$ are shown in Figure 4 Unfortunately, a new curve-fit must be generated for each vehicle geometry and $\beta_{2} / \beta_{1}$, as the optimal jettison time is heavily dependent on these parameters.

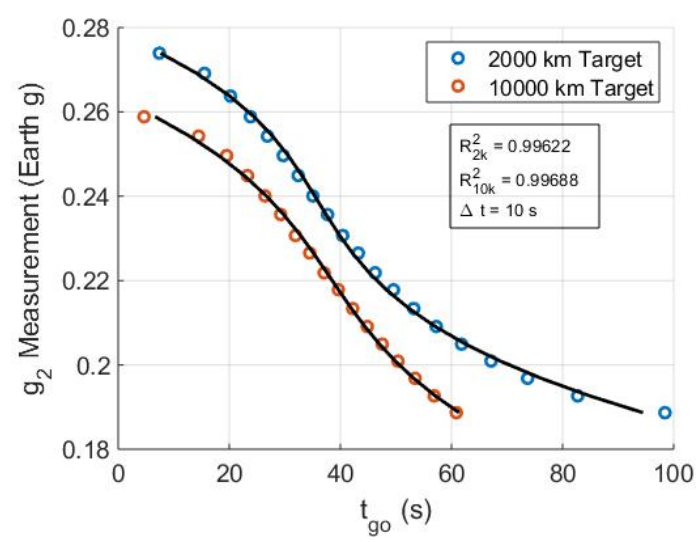

(a) Deceleration Curve-Fit as Function of Target Apoapsis, $\beta_{2} / \beta_{1}=9.18$

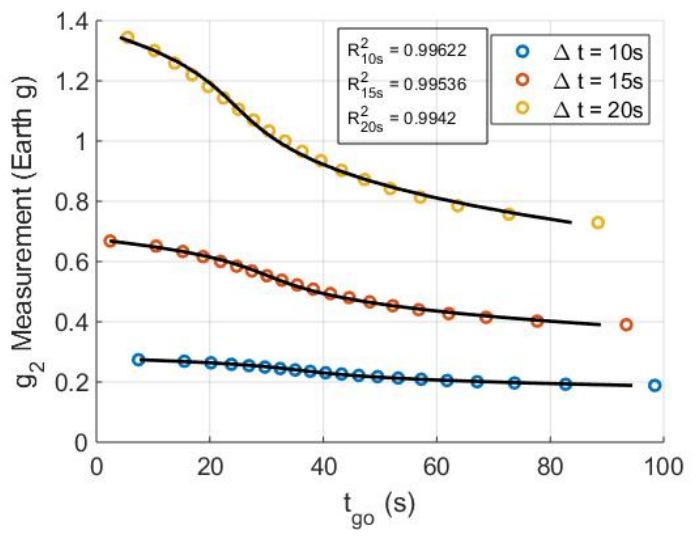

(b) Deceleration Curve-Fit as Function of $\Delta t$ Values, $\beta_{2} / \beta_{1}=9.18$

Fig. 4 Deceleration Curve-Fits for Apoapsis Targets and Measurement $\Delta t$ Values

The $R^{2}$ values for each curve are promisingly high, although the curve fit still falls above and below the actual $g_{2}$ measurement values for various EFPAs, which may cause nominal jettison time errors and subsequent high apoapsis errors.

This guidance algorithm is extremely computationally efficient because the deceleration curve quadratic constants are pre-determined. In addition, the guidance call rate can be very low, as the jettison time is only determined once from the curve fit data. This unfortunately means that this algorithm is vulnerable to perturbation errors; an off-nominal deceleration measurement will result in a large jettison time error. This may lead to significantly large apoapsis altitude errors, or even failure to exit the atmosphere if the jettison time occurs too late in the trajectory.

To mitigate this error, a deceleration corrector is introduced for dispersion analysis; because the deceleration curve-fit is pre-generated, the nominal deceleration measurement value is known for a given EFPA; this can be implemented as an input to the guidance system to develop a decelerator corrector, $\frac{g_{2}}{g_{2, \text { nom }}}$, which is then multiplied by the in-flight $g_{2}$ measurement when determining the jettison time from the curve fit quadratic constants. 


\section{Predictive Guidance Algorithm}

A much simpler form of the NPC algorithm introduced in Section II.C.3 seeks to remove the corrector term entirely; this predictive guidance algorithm solely propagates the current state forward in time from the current state if jettison were to occur at the current time step. This allows for various parameter triggers to determine when jettison should occur. The development of a PDG algorithm for ballute jettison by Miller et. al. used an energy trigger that compared the orbital energy of the vehicle to the desired orbital energy [9]. The desired orbital energy can be computed from a target apoapsis and the minimum altitude of the propagated state, assuming the vehicle succeeds in skipping out of the atmosphere instead of landing on the surface.

The orbital energy trigger is applied to this guidance system, where jettison occurs when $\zeta_{\text {curr }}-\zeta_{\text {des }}<=0 . \zeta_{\text {curr }}$ is calculated at each guidance function call by propagating the current state forward in time using second-stage vehicle parameters. The performance of this guidance system is thus highly dependent on the guidance call rate because the lack of a corrector term means jettison can only occur on a guidance function time step. So, while the algorithm itself is less computationally expensive than an NPC, the overall computational requirement is still high, as the guidance integration rate and guidance call rates must be high enough to prevent biasing error as discussed in Section II.C.4.

A density corrector is implemented for dispersion analysis just as in the NPC algorithm described in Section II.C.3. this mitigates against day-of-flight atmospheric uncertainties, although it is still incapable of correcting against post-jettison uncertainties for single-stage jettison systems.

\section{Numerical Predictor-Corrector Algorithm}

The original numerical predictor-corrector algorithm written by Putnam and Braun implemented a bisection method to reduce the apoapsis altitude error to zero [10]. The guidance function propagated the current vehicle state to atmospheric exit given nominal atmospheric knowledge and a jettison time estimate to reduce the apoapsis altitude error using a corrector function. The bisection method is a robust method that requires the solution to be bounded; unfortunately, it is also quite slow, particularly for poor initial jettison time estimates. The original algorithm included an iterative function, where the guidance algorithm would iterate on the jettison time up to 5 times per guidance function call. This allowed the guidance function to determine a jettison time in a few guidance function calls; however, it also increases the computational cost drastically. The benefits of using this internal iterative solution method is that the guidance function can adjust to current atmospheric density variation estimates within a single guidance call from the density corrector term, rather than constantly needing to update its estimate of the atmosphere at each guidance call.

In an effort to improve the solution speed of this algorithm, the predictor-corrector is restructured with a numeric Newton-Raphson root-finding method. The function of interest is the apoapsis altitude error, in which the derivative is calculated by adjusting the jettison time by a small increment to numerically compute the function derivative, as shown in Equation 2. While the Newton method is promising in terms of computational efficiency, it can become unstable due to the lack of solution bounds. This can be guarded against by providing a good initial jettison time estimate as with the bisection method, allowing the method to converge very quickly for reasonable uncertainties in the atmosphere and vehicle state.

$$
\begin{array}{r}
x_{i+1}=x_{i}-\frac{d r_{a} / d t_{j}}{\Delta r_{a}} \\
\frac{d r_{a}}{d t_{j}} \approx \frac{\Delta r_{a}}{\Delta t_{j}}
\end{array}
$$

The Newton method can also be very unstable if the update term is very large due to a poor initial guess or large change in atmospheric density. This is guarded against for cases where the current jettison time estimate produces either a hyperbolic exit trajectory or inability to exit the atmosphere by bounding the update term by a fixed value. This way, if the update term is too large, the Newton method will iterate by some fixed increment towards the optimal solution instead of overshooting it by some unrealistic time change.

Finally, the guidance integration rate must be sufficiently large such that the jettison time change accurately represents a function derivative. If the $\Delta t$ value is too high, the nonlinearity of the convergence function will cause a biasing error in the guidance estimate. This further increases the computational requirement of the NPC algorithm, while removing biasing errors in the process. 


\section{Guidance Algorithm Error Mitigation}

The guidance, navigation, and control systems are decoupled from the main integrator function in an effort to simulate a real flight system that may not be able to perform guidance calculations at $10 \mathrm{~Hz}$ or more. This also enables the user to analyze the performance of a guidance algorithm based on the call rate as a primitive supplement for quantifying computational cost.

Various differences between guidance and simulation models can cause large biasing errors in the desired final state. More specifically, the guidance model simplicity plays a large factor in the algorithm performance; if the dynamics and/or gravity models are over-simplified, then the algorithm will be propagating a state different than the actual trajectory would follow, causing it to solve a slightly different optimization problem. Similarly, the guidance integration rate will cause a biasing error if it is too low compared to the simulation rate. This is due to large time step numerical errors propagating to atmospheric exit, resulting in the different final estimated state.

In order to visualize these errors, Figure 5 below depicts the apoapsis altitude error between a simplified guidance model and a more complex one. It depicts nominal apoapsis errors using an NPC algorithm over a range of EFPAs. Figure 5 a uses an integration rate of $10 \mathrm{~Hz}$, calculating dynamics with only aerodynamics and inverse-square gravity, and neglecting planet rotation or planet-relative motion. It is clear that, while a shallower EFPA results in a relatively low error, the apoapsis error increases rapidly for a steeper EFPA, even given perfect knowledge about the atmospheric properties and vehicle state. Given that Venus is a difficult target to perform aerocapture as detailed in Section [] dispersion analysis with this large of a biasing error would result in very few trajectories that attain the desired apoapsis altitude.

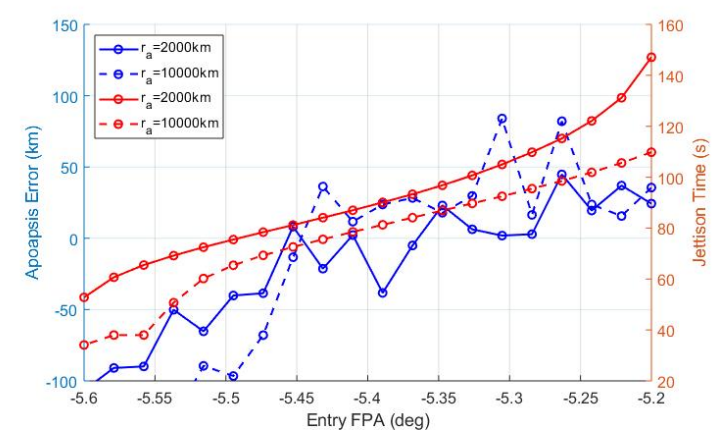

(a) Jettison Times and Nominal Apoapsis Errors vs. EFPA for Biased Guidance System

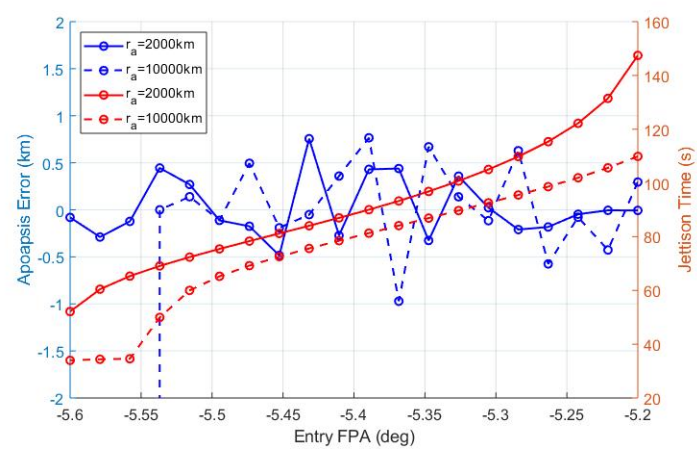

(b) Jettison Times and Nominal Apoapsis Errors vs. EFPA for Unbiased Guidance System

\section{Fig. 5 Optimal Jettison Times and Nominal Apoapsis Errors vs. Entry Flight Path Angle for different Guidance Models}

For comparison, Figure $5 \mathrm{~b}$ shows the nominal errors for a range of EFPAs if the simulation and guidance models and integration rates match precisely. All of the apoapsis errors are consistently below $1 \mathrm{~km}$, indicating that the guidance algorithm is unbiased and working properly, as any reasonable EFPA should yield an exact jettison time to drive the apoapsis error to zero with perfect knowledge of the system. Note that the sudden increase in apoapsis error for a $10000 \mathrm{~km}$ target altitude at around $-5.5^{\circ}$ is due to the optimal jettison time occurring before the vehicle has reached a sensible atmosphere to start performing guidance iterations. This bound on EFPA is determined by the vehicle ballistic coefficient ratio, as well as the target apoapsis.

\section{Density Corrector Performance}

In addition to guidance model bias errors, the density corrector performance is tied to the guidance call rate. Figure 6 depicts the density corrector multiplier value against the true disperse atmospheric density variation as a function of altitude for different guidance call rates. The density corrector is only utilized inside the guidance function, meaning that it is only calculated with each guidance function call; the lower the guidance call rate, the less accurate the density corrector term will be due to its dependence on previous corrector values, as shown in Equation 1.

While the benefits of a higher-fidelity guidance system are evident, the complexity of the model will increase the computational cost of the system, reducing the number of feasible guidance function calls until jettison must occur. This 


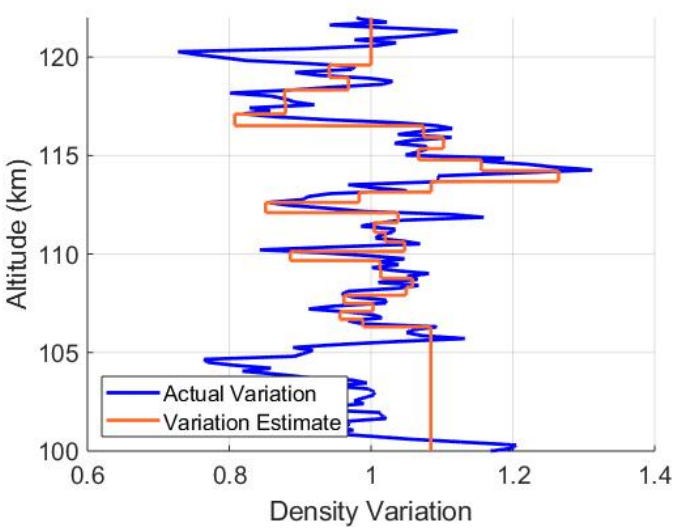

(a) Density Corrector Performance, Guidance Rate $1 \mathrm{~Hz}$

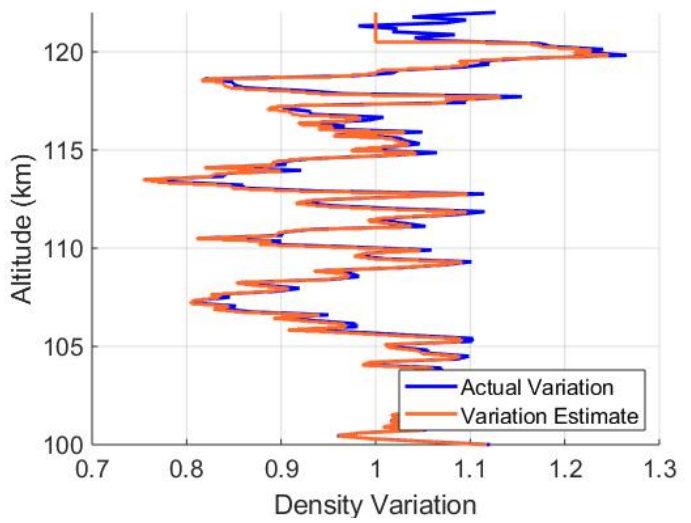

(b) Density Corrector Performance, Guidance Rate $10 \mathrm{~Hz}$

Fig. 6 Density Corrector Performance as function of Guidance Call Rate (Hz)

is particularly daunting for vehicles that enter the atmosphere at steep EFPAs, as the jettison times for these initial states is typically very early on in the trajectory, as highlighted in Figure 5 This inherent trade-off between biasing error and computational cost is a large driving factor for the feasibility of aerocapture at planetary bodies. Improvements for the density corrector are discussed in Section $\mathrm{V}$.

\section{Results}

\section{A. EFPA Trade Studies}

Before beginning to develop and analyze various guidance algorithms, EFPA trade studies were performed concerning aerocapture at Venus. This was done to understand the limitations and constraints of the particular problem.

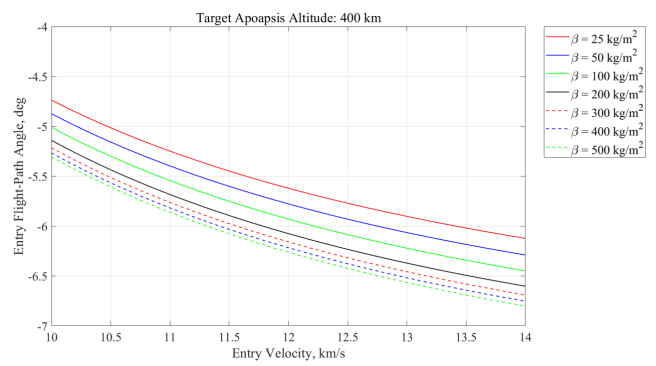

(a) Entry Flight Path Angle vs. Entry Velocity for Various $\beta$ 's Targeting $400 \mathrm{~km}$ Apoapsis Altitude

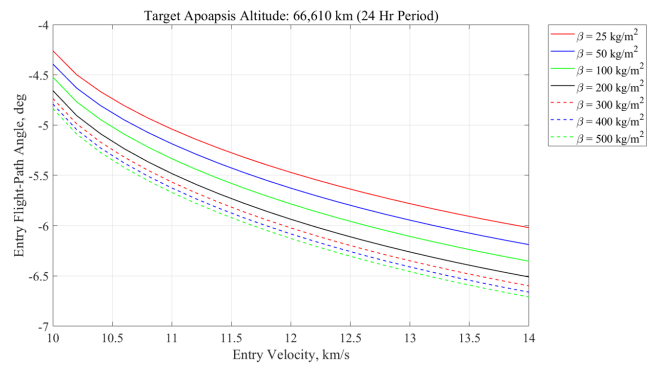

(b) Entry Flight Path Angle vs. Entry Velocity for Various $\beta$ 's Targeting $24 h r$ Orbital Period

\section{Fig. 7 Apoapsis Error vs. Guidance Function Calls for Different Root-Finding Methods}

Figure 7 plots EFPA vs. entry velocity to target both a $400 \mathrm{~km}$ apoapsis altitude and a $24 \mathrm{hr}$ orbital period for a variety of ballistic coefficients. These entry trades do not consider any control authority; instead, they highlight how the required EFPA changes for a given entry velocity at Venus to reach a specific orbit. Figure 7 indicates that a smaller ballistic coefficient or lower entry velocity allows the EFPA to be shallower, which has strong implications for the probability of capture as related to the optimal jettison time.

Figure 8 details how the entry flight-path-angle corridor width changes as a function of ballistic coefficient ratio, $\beta_{2} / \beta_{1}$, for various entry velocities targeting an apoapsis altitude of $400 \mathrm{~km}$. It is evident that the corridor is relatively insensitive to entry velocity, although it does widen with increasing $\beta$ ratio. This constrains the interplanetary trajectory targeting systems, although the widths shown in Figure 8 are not unreasonable. 


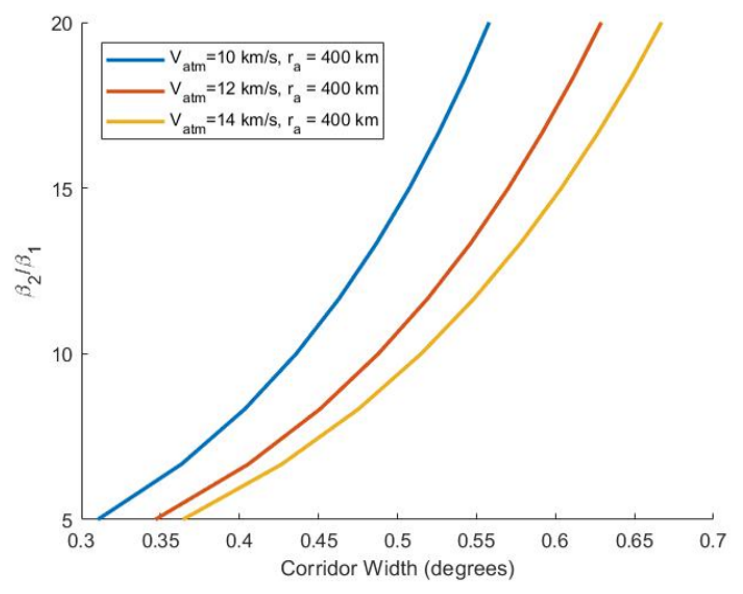

Fig. $8 \beta_{2} / \beta_{1}$ Ratio vs. EFPA Corridor Width

\section{B. Deceleration Curve-Fit Algorithm}

Figure 9a below shows the nominal apoapsis altitude error as a function of EFPA for various $g_{2}$ measurement $\Delta t$ values, while Figure $9 \mathrm{~b}$ shows the difference in nominal jettison times between the DCF and the unbiased NPC jettison times from Figure $5 b$

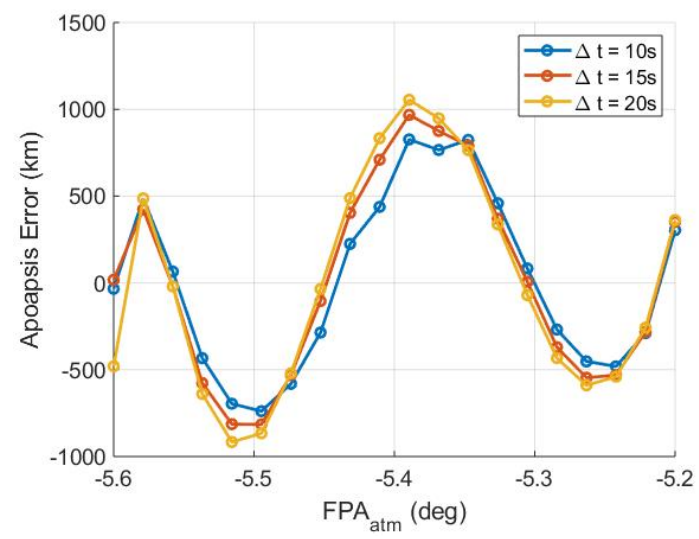

(a) Nominal Apoapsis Error vs. EFPA for various $g_{2}$ measurement $\Delta t \mathrm{~s}$

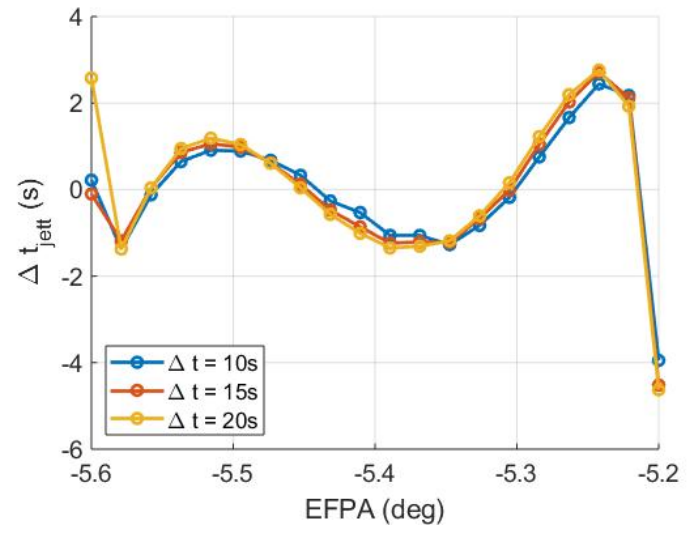

(b) Nominal Jettison Time Difference between DCF and NPC Algorithms

Fig. 9 Nominal Deceleration Curve-Fit Error and Jettison Time Results for $\beta_{2} / \beta_{1}=9.18$ and $h_{a, t g t}=2000 \mathrm{~km}$

The trend in the apoapsis altitude errors matches with those reported in Werner and Braun, although the error values themselves are much higher than desired [8]. The errors are related to the accuracy of the quadratic curve fit; if the curve fit predicts a greater deceleration than the actual measurement, the jettison time will occur earlier, yielding an apoapsis that is above the target. Likewise, a curve fit that predicts a lower deceleration will result in an apoapsis altitude below the target, yielding in the sinusoidal-like trend in Figure 9a Only 15\% of nominal cases fall within $100 \mathrm{~km}$ of the target apoapsis for $2000 \mathrm{~km}$. These large errors are caused by jettison time differences of less than 2 seconds for most EFPAs, highlighting the argument for the difficulty of performing aerocapture at Venus discussed in Section I.B.

Figure 10 shows apoapsis altitude errors and jettison time differences as functions of EFPA for different target apoapsis altitudes. The large errors are caused by the nonlinearity in the deceleration curves depicted in Figure 4. This nonlinearity causes the curve fit to fall slightly off of the true deceleration measurements, yielding deceleration estimate errors corresponding to the shown apoapsis errors in Figures 9a and 10a. Interestingly enough, the difference target apoapsis yields opposing error trends, as shown in Figure 10a These dissimilar trends are caused by the curve fit falling above vs. below the nominal deceleration measurement for a given EFPA. 


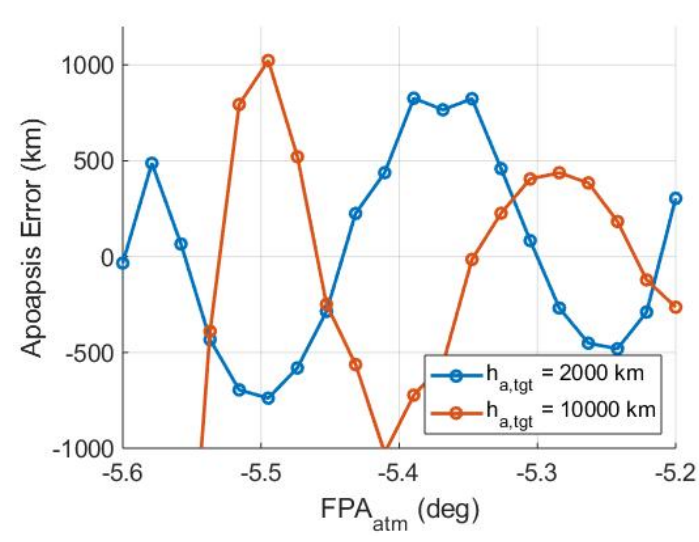

(a) Nominal Apoapsis Error vs. EFPA for various Apoapsis Altitude Targets

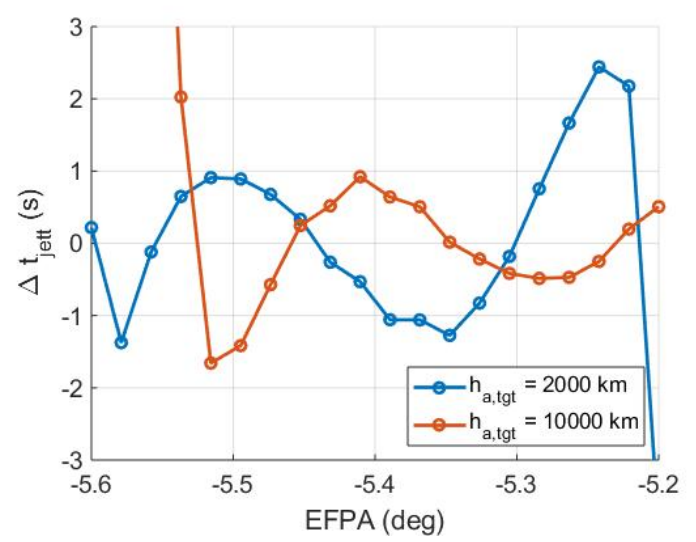

(b) Nominal Jettison Time Difference between DCF and NPC Algorithms

Fig. 10 Nominal Deceleration Curve-Fit Error and Jettison Time Results for $\beta_{2} / \beta_{1}=9.18 \Delta t=10 \mathrm{~s}$

To assess the performance under uncertainty, Figure 11 shows shows a set of Monte Carlo simulations for various EFPAs and target apoapsis altitudes. The apoapsis errors tend to be skewed to the left, a direct result of the deceleration corrector modifying the measured deceleration to fall below the expected deceleration value. This matches the expected negative apoapsis altitude error reported in Figure 10 for the given EFPA of $-5.5^{\circ}$. Even so, the capture rate targeting $2000 \mathrm{~km}$ is greater than $55 \%$ within $1000 \mathrm{~km}$ for both EFPAs.

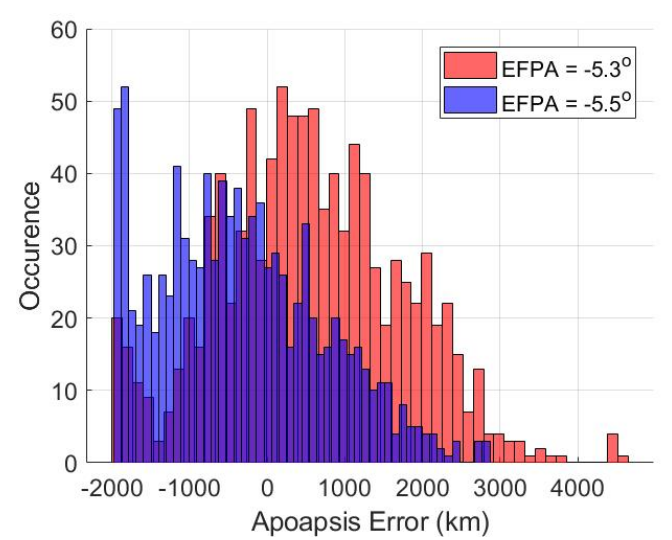

(a) Monte Carlo Apoapsis Error Results for different EFPAs and $h_{a, t g t}=2000 \mathrm{~km}$

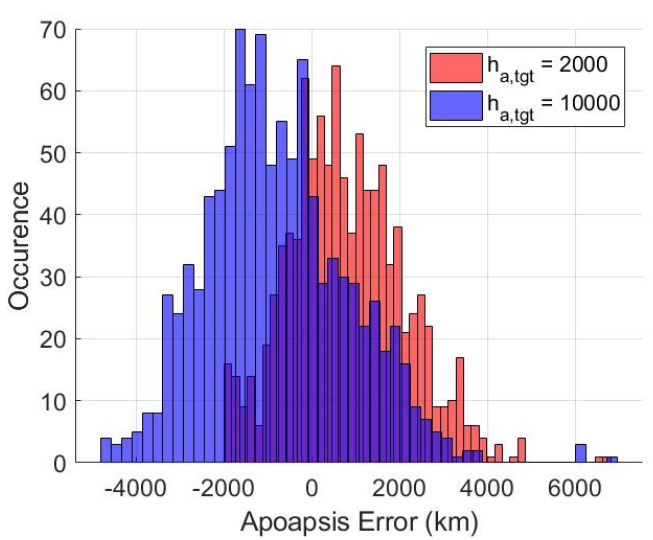

(b) Monte Carlo Apoapsis Error Results for different Apoapsis Targets and $E F P A=-5.4^{\circ}$

Fig. 11 Monte Carlo Results using Deceleration Curve-Fit Algorithm for Various EFPAs and Target Altitudes

The lower altitude target has a minority of cases impacting the surface of Venus; however, the variance in apoapsis errors is not as large as the higher apoapsis altitude case shown in Figure 11b. Given the trend observed in Figure 11a, a shallower EFPA might further reduce the percentage of surface impacts for a low apoapsis altitude target at the risk of exiting the atmosphere on a hyperbolic trajectory if insufficient energy is lost.

\section{Predictive Guidance Algorithm}

The following section highlights the results of the pure prediction algorithm derived from the energy-trigger ballute aerocapture paper by Miller et. al. [9]. Different trajectory integration and guidance call rates are implemented to highlight the performance as a function of computational cost.

Figure 12 shows the nominal apoapsis altitude errors for different guidance call rates using the predictive guidance 
algorithm outlined in Section II.C.2 Both Figures $12 \mathrm{a}$ and $12 \mathrm{~b}$ are integrated at $100 \mathrm{~Hz}$ targeting both $2000 \mathrm{~km}$ and $10000 \mathrm{~km}$ apoapsis altitudes, with guidance call rates of $10 \mathrm{~Hz}$ and $5 \mathrm{~Hz}$, respectively. Note that the large increase in error for the $10000 \mathrm{~km}$ target apoapsis cases for EFPAs steeper than $-5.5^{\circ}$ is again due to the optimal jettison time occurring before the vehicle has reached a sensible atmosphere.

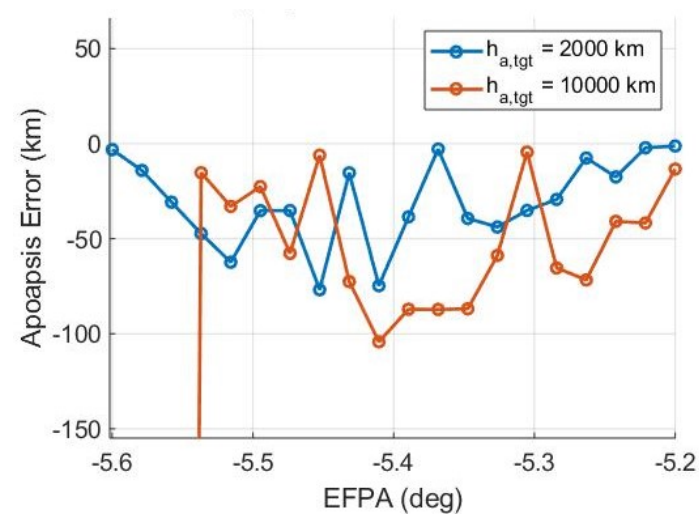

(a) Nominal Apoapsis Error vs. EFPA at $10 \mathrm{~Hz}$ Guidance Rate

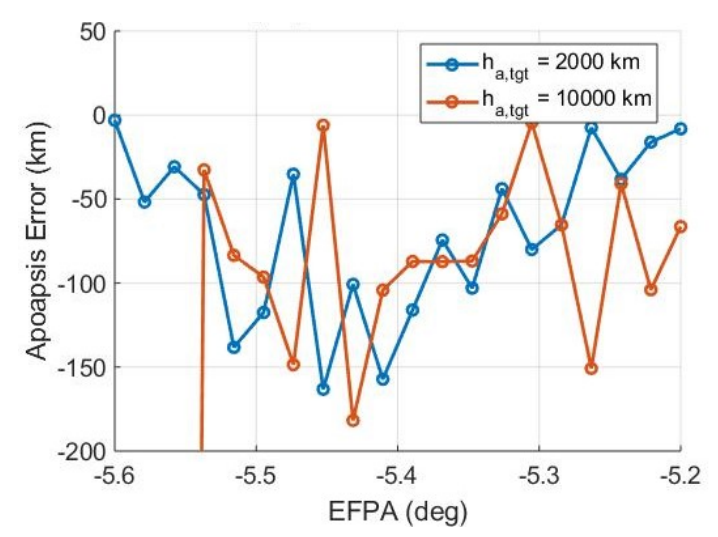

(b) Nominal Apoapsis Error vs. EFPA at $5 \mathrm{~Hz}$ Guidance Rate

Fig. 12 Nominal Apoapsis Error vs. EFPA using PDG algorithm, $\beta_{2} / \beta_{1}=9.18$ and Integration Rate of $100 \mathrm{~Hz}$ at Two Different Target Apoapsis Altitudes

As mentioned in Section II.C.2 jettison can only occur on a time step in which the guidance function is called due to the absence of a corrector function. This means the guidance algorithm performance is heavily coupled with the guidance call rate, as evidenced by Figure 12 With a guidance call rate of $10 \mathrm{~Hz}$, all of the nominal errors are below $100 \mathrm{~km}$, wheres a guidance call rate of $5 \mathrm{~Hz}$ causes the errors to grow above $150 \mathrm{~km}$ nominally, as well as cause much larger variations in errors between EFPAs data points.

These error variations can be explained by the inherent coupling of the jettison command with the guidance call rate; A guidance rate of $5 \mathrm{~Hz}$ means that the jettison time can only occur in segments of 0.2 seconds, whereas the accuracy of the optimal jettison time might be on the order of thousandths of a second. The nonlinearity of the optimal jettison time curve, shown in Figure 5b explains how the linear time step increase of the guidance function call causes the large variability in apoapsis errors between EFPA data points. If the guidance call rate were large enough, the apoapsis errors would drop to zero in all cases, as the guidance system would have knowledge of the predicted state at every major time step; however, this is unreasonable as the state propagation within the guidance algorithm is computationally costly and cannot be physically run at such a high frequency.

It should also be noted that all of the nominal errors in Figure 12 are less than 0, a consequence of the predictive guidance algorithm only jettisoning when the orbital energy is less than or equal to the desired value. A tolerance on orbital energy could be implemented to allow jettison within the specified tolerance to allow cases to fall above the target apoapsis; however, a tolerance on orbital energy is somewhat unintuitive.

Figure 13 depicts the Monte Carlo analysis results of a vehicle with $\beta_{2} / \beta_{1}=9.18$, integrated at $100 \mathrm{~Hz}$ and a guidance rate of $5 \mathrm{~Hz}$. Figure $13 \mathrm{a}$ displays the dispersion analysis statistics for two different apoapsis altitude targets, while Figure $13 \mathrm{~b}$ assesses the performance of the guidance algorithm for three different EFPAs.

It is evident from Figure 13 a that the algorithm performs better for lower altitude targets, a trend also somewhat apparent from Figure 12. This is a direct result of the optimal jettison time occurring earlier in the trajectory for a higher apoapsis altitude, allowing a larger time frame in which the vehicle is vulnerable to atmospheric uncertainties. However, the apoapsis errors for the $2000 \mathrm{~km}$ target case extend to $-2000 \mathrm{~km}$, indicating that a small minority of trajectories impacted the surface, while the $10000 \mathrm{~km}$ target simulation has zero cases impacting the surface, even though the variation in errors is much larger.

Figure $13 \mathrm{~b}$ shows an increased apoapsis altitude error for steeper EFPAs, a trend not shown in the nominal error cases. The shallowest case, an EFPA of $-5.2^{\circ}$, has apoapsis errors contained entirely within $\pm 500 \mathrm{~km}$. The trend of increasing apoapsis error with EFPA steepness can be explained again by the earlier jettison times increasing vehicle vulnerability to downrange atmospheric uncertainties. More specifically, $50 \%$ of trajectories successfully captured within $100 \mathrm{~km}$ for an EFPA of $-5.2^{\circ}$ targeting $10000 \mathrm{~km}$, and over $90 \%$ of cases captured within $250 \mathrm{~km}$ at this same 


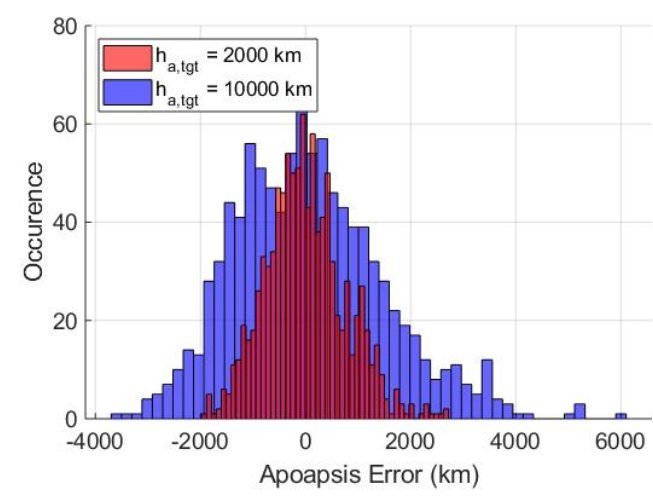

(a) PDG Monte Carlo Results for Different Apoapsis Altitude Targets, EFPA $=-5.4^{\circ}$

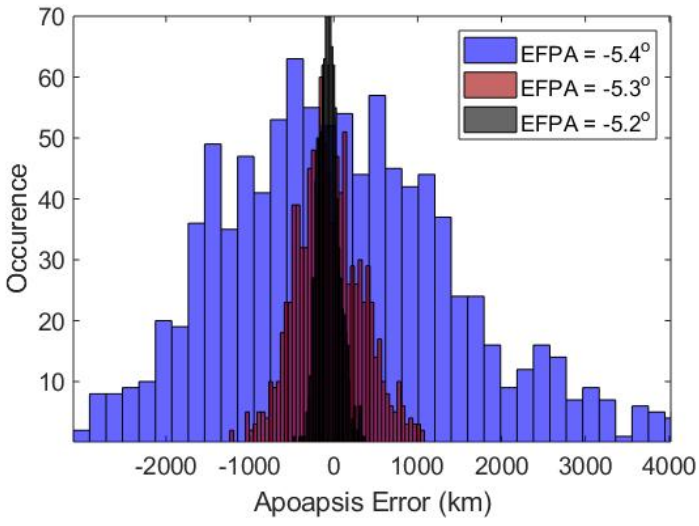

(b) PDG Monte Carlo Results for Various EFPAs Targeting $10000 \mathrm{~km}$

Fig. 13 PDG Monte Carlo Results for Different Apoapsis Altitude Targets and EFPAs, Integrated at $100 \mathrm{~Hz}$ and Guidance Rate $=5 \mathrm{~Hz}$ with $\beta_{2} / \beta_{1}=9.18$

entry state and target altitude.

\section{Numerical Predictor-Corrector Algorithm}

As discussed in Section II.C.3 the NPC algorithm was restructured to allow iteration using either a bisection method or a Newton method to converge on an optimal jettison time. Figure 14 below shows the solution comparison between the Newton method and the bisection method for two different guidance call rates using the same simulation inputs for a nominal case with a vehicle consisting of a ballistic coefficient ratio of $\beta_{2} / \beta_{1}=9.18$.

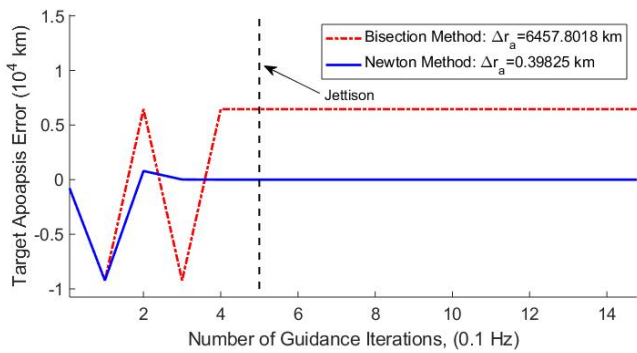

(a) Nominal Apoapsis Error vs. Guidance Function Calls at $0.1 \mathrm{~Hz}$

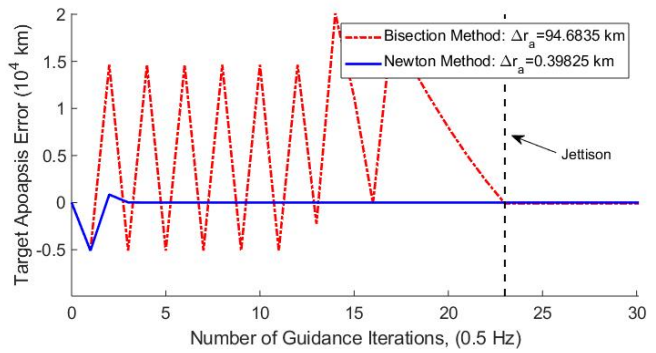

(b) Nominal Apoapsis Error vs. Guidance Function Calls at $0.5 \mathrm{~Hz}$

Fig. 14 Apoapsis Error vs. Guidance Function Calls for Different Root-Finding Methods Targeting 10000km Apoapsis Altitude

Note that here the number of internal iterations performed by each guidance algorithm is set to 1 to output every iteration of the solution for visualization of the guidance law mechanics. Not only does Figure 14 highlight the solution speed improvement of the Newton method, it also highlights the relative sensitivity of the initial guess. Figure $14 \mathrm{~b}$ shows that the bisection method requires over 20 iterations to converge, still producing an error of almost $100 \mathrm{~km}$ with perfect atmospheric and state knowledge, while the Newton method converges on an error of less than $1 \mathrm{~km}$ in fewer than 5 updates.

Figure 15 shows capture percentage vs. apoapsis altitude error tolerance for a variety of ballistic coefficient ratios using the Newton root-finding method. Each data point is a separate Monte Carlo simulation with the NPC algorithm run at $5 \mathrm{~Hz}$ and an overall simulation integration rate of $100 \mathrm{~Hz}$. Small changes in $\beta_{2} / \beta_{1}$ ratios do not impact the capture rate significantly, although large increases in $\beta_{2} / \beta_{1}$ ratios appear to increase the capture rate by $10 \%-20 \%$ for a given apoapsis altitude tolerance. This has strong implications for aerocapture vehicle architecture design. In general, 


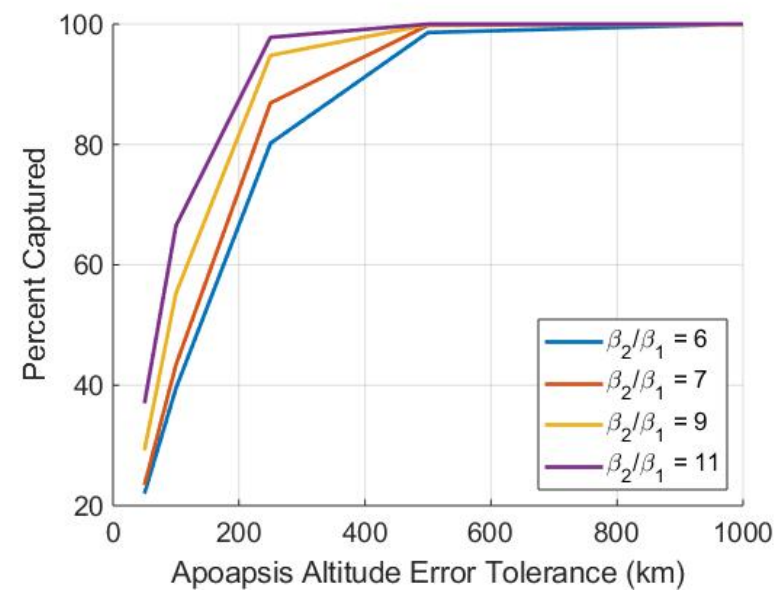

Fig. 15 Capture Rate vs. Apoapsis Altitude Tolerance for Various $\beta_{2} / \beta_{1}$ Ratios with Newton Method Root-Finder at a GNC Rate of $5 \mathrm{~Hz}$, Targeting a $10000 \mathrm{~km}$ Apoapsis Altitude

architectures with a $\beta_{2} / \beta_{1}$ ratios above 7 achieved a capture rate of above $50 \%$ within $100 \mathrm{~km}$ of a $10000 \mathrm{~km}$ target apoapsis altitude, while all $\beta_{2} / \beta_{1}$ ratios captured within $250 \mathrm{~km}$ at a rate of $80 \%$. The largest of these, a $\beta_{2} / \beta_{1}$ ratio of 11 , reached a capture rate above $97 \%$ within $250 \mathrm{~km}$. Increasing the tolerance further resulted in all $\beta_{2} / \beta_{1}$ ratios capturing $99.9 \%$ of the time within $1000 \mathrm{~km}$ when targeting $10000 \mathrm{~km}$.

\section{NPC Method Comparison}

This section seeks to showcase the performance of the Newton-Raphson method when compared to the bisection method within the NPC algorithm. Figure 16 shows the Monte Carlo analysis results for each root-finding method for a vehicle with $\beta_{2} / \beta_{1}=9.18$, entering with an EFPA of $-5.4^{\circ}$, and targeting $10000 \mathrm{~km}$ apoapsis altitude at different guidance frequencies. The guidance rates, $0.1 \mathrm{~Hz}$ and $1 \mathrm{~Hz}$ respectively, used in Figure 16 are more realistic for a flight scenario, and seek to highlight the performance of each root-finding method in a more practical setting.

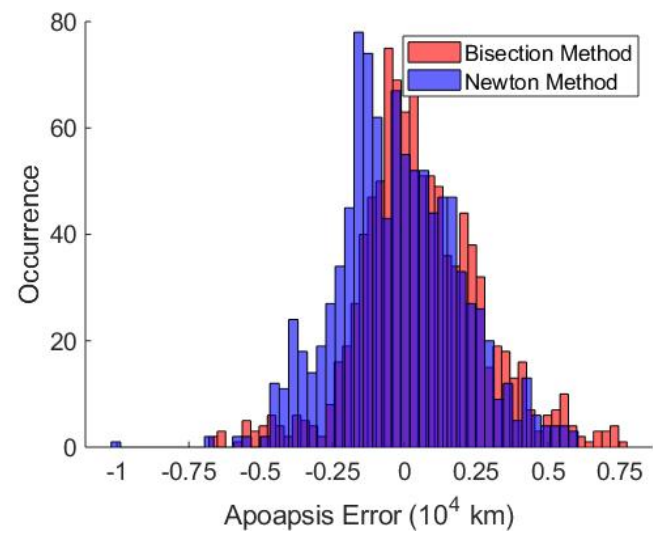

(a) Apoapsis Error vs. Guidance Function Calls at $1 H z$

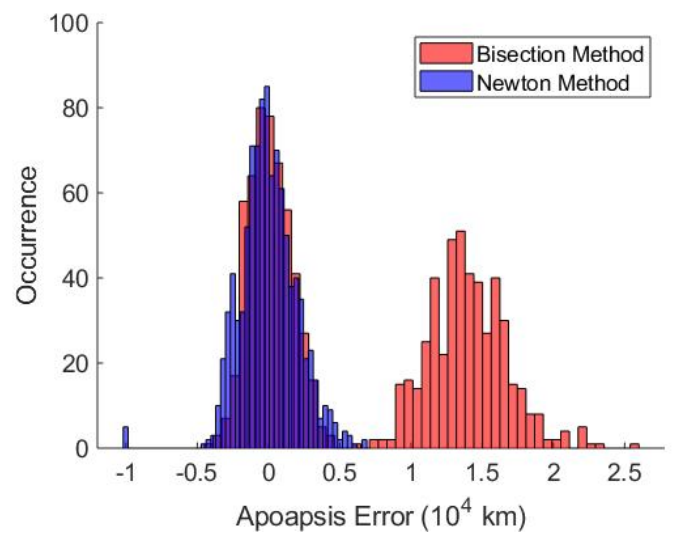

(b) Apoapsis Error vs. Guidance Function Calls at $0.1 \mathrm{~Hz}$

Fig. 16 NPC Monte Carlo Results Comparing Root-Finding Method Performance at Various Guidance Call Rates, EFPA $=-5.4^{\circ}, \beta_{2} / \beta_{1}=9.18$, Apoapsis Altitude Target of $10000 \mathrm{~km}$, and Max Corrector Iteration of 1

Figure 16 shows that the Newton method is a more robust jettison time update solution, causing the vehicle to capture into an orbit instead of impacting the surface in almost every case, regardless of guidance call frequency. In contrast, Figure $16 \mathrm{~b}$ shows that the bisection method is too slow to converge on a solution in roughly $40 \%$ of cases that 


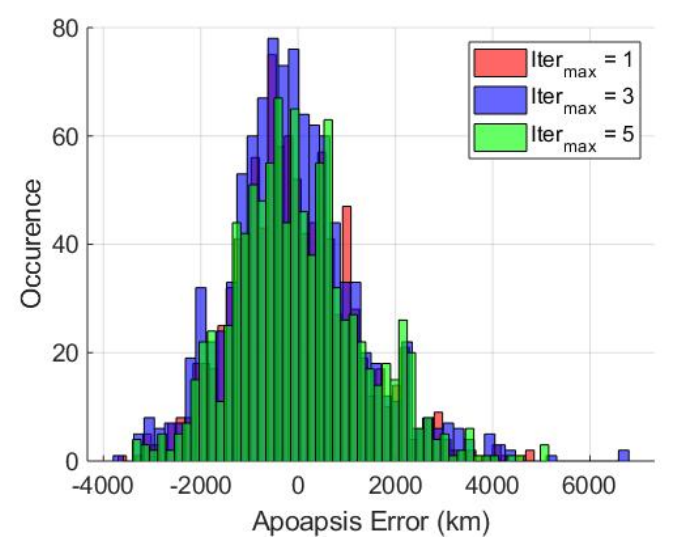

(a) Monte Carlo NPC Results for Newton Method as Function of Internal Iterations

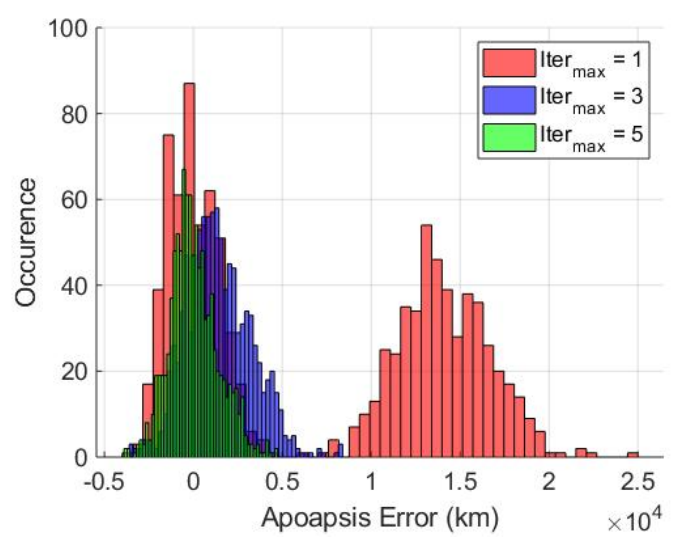

(b) Monte Carlo NPC Results for Bisection Method as Function of Internal Iterations

Fig. 17 Monte Carlo Results for Different NPC Method as Function of Internal Iterations Targeting 10000km, $\beta_{2} / \beta_{1}=9.18$, Guidance Rate of $0.1 \mathrm{~Hz}$, and Integration Rate of $100 \mathrm{~Hz}$

target more than double the desired apoapsis altitude for a given initial jettison time estimate of $85 \mathrm{~s}$, only a few seconds off of the nominal optimal jettison time shown in Figure $5 \mathrm{~b}$ for the same entry conditions and apoapsis target. However, Figure 16a shows that, when the bisection method has sufficient time to find a solution, it will perform at least as well as the Newton method, if not better. This is likely due to the instability of the Newton method caused by changing atmospheric density between guidance calls.

Figure 17 depicts the performance of the two root-finding methods as a function of the internal number of iterations, simulated with a guidance rate of $0.1 \mathrm{~Hz}$ and a simulation integration rate of $100 \mathrm{~Hz}$. It is clear that an increased number of internal iterations has a negligible effect on the Newton method while slowly shifting the median apoapsis error towards zero for the bisection method.

Both root-finding schemes are able to deliver a payload into orbit about Venus with $100 \%$ success targeting $10000 \mathrm{~km}$ apoapsis altitude and a tolerance of over $5000 \mathrm{~km}$; however, for a low guidance rate the bisection method has a high probability of cases that capture into orbit at up to 2 times the target apoapsis, a trend that is likely worsened for lower apoapsis targets.

\section{Conclusion}

This paper investigates the performance of three different guidance algorithms applied to performing a singular drag skirt separation event for drag-modulated aerocapture at Venus; the three algorithms are a deceleration curve-fit algorithm, a pure-state prediction algorithm, and a numerical predictor-corrector algorithm using both bisection and Newton root-finding methods. Venus is arguably the most difficult planetary body in the solar system to perform aerocapture at due to the high atmospheric density and temperature. If drag-modulated aerocapture can be proven feasible at Venus, it should therefore be feasible at most, if not all, other planetary bodies in the solar system. The major contributor to the errors at Venus are the large apoapsis altitude errors caused by small errors in jettison due to the high atmospheric density. The three algorithms were analyzed using a 4th-order Runge-Kutta integrator developed in Matlab and atmospheric data tabulated from VenusGRAM. Monte Carlo simulations were performed to assess guidance law performance under uncertainty.

It was shown that the deceleration curve-fit was able to achieve $>55 \%$ capture rate targeting a $2000 \mathrm{~km}$ apoapsis altitude with a tolerance of $1000 \mathrm{~km}$ for both EFPAs of $-5.3^{\circ}$ and $-5.5^{\circ}$. In addition, lower altitude apoapsis targets increased guidance law performance due to the precision of the curve fits for these altitudes. The deceleration curve-fit algorithm is also the least computationally costly of the three algorithms analyzed.

The predictive algorithm achieved a $50 \%$ capture rate with an EFPA of $-5.2^{\circ}$ targeting a $10000 \mathrm{~km}$ apoapsis altitude within $100 \mathrm{~km}$, and $>90 \%$ within $250 \mathrm{~km}$. This was done with a guidance rate of $5 \mathrm{~Hz}$, which limited the accuracy of the jettison time to segments of 0.2 seconds. Shallower EFPAs and lower apoapsis altitude targets increased the algorithm performance due to the delayed jettison time. The computational cost of this algorithm can be prohibitively expensive 
due to the constraint of jettison timing occurring on a guidance call time step.

Finally, the numerical predictor-corrector algorithm demonstrated a trend of increasing capture rate as a function of ballistic coefficient ratio. $\beta_{2} / \beta_{1}$ ratios greater than 7 achieved $>50 \%$ capture rate for a target apoapsis altitude of $10000 \mathrm{~km}$ within $100 \mathrm{~km}$, while all considered $\beta_{2} / \beta_{1}$ ratios attained $>80 \%$ capture rate within $250 \mathrm{~km}$ with the largest of the ratios achieving $>97 \%$ success. All considered ratios achieve $99.9 \%$ capture within $1000 \mathrm{~km}$ of the target apoapsis of $10000 \mathrm{~km}$ using the Newton root-finding method. All of the algorithms observed similar trends in terms of algorithm performance as a function of EFPA and apoapsis altitude target due to the jettison time and resulting vulnerability to downstream uncertainty. However, the NPC algorithm was able to achieve a $99.9 \%$ capture rate within a tolerance of $10 \%$ of the target altitude.

\section{Future Work}

It is likely that a planet with a lower atmospheric density would achieve similar capture rates under tighter apoapsis constraints. Given that the largest source of error was atmospheric uncertainty downstream of the jettison event, multiple jettison events should be assessed to mitigate downrange errors to improve performance. The NPC algorithm would be ideal for multiple jettison events if implemented such that it could iterate on each stage during each guidance call, rather than needing to "restart" after each separation event. For the PDG algorithm, other jettison triggers such as altitude might enable a more intuitive trigger tolerance, allowing for a broader range of jettison times given that the resultant apoapsis altitude decreases monotonically with jettison time.

Finally, future work should analyze system performance at a multitude of planetary bodies, rather than focusing on a single one. This might provide insight as to the feasibility of this architecture at specific planetary bodies as opposed to proving it for the hardest possible case. The lower atmospheric density at other bodies should theoretically improve capture rates for a given apoapsis altitude tolerance due to smaller errors caused by jettison time differences.

\section{Acknowledgements}

The authors would like to thank the Jet Propulsion Laboratory for funding this research during the 2017-2018 Fiscal Year.

\section{References}

[1] Braun, R. D., "Planetary Entry, Descent, and Landing Shortcourse," International Planetary Probe Workshop, Pasadena, CA, 2014.

[2] Hall, J. L., Noca, M. A., and Bailey, R. W., "Cost - Benefit Analysis of the Aerocapture Mission Set,” Vol. 42, No. 2, 2005.

[3] Powell, R., and Braun, R., "A Six-Degree-of-Freedom Guidance and Control Analysis of Mars Aerocapture,” 30th Aerospace Sciences Meeting and Exhibit, Vol. 16, No. 6, 1992, pp. 1038-1044. doi:10.2514/6.1992-736, URL http://arc.aiaa.org/ doi/10.2514/6.1992-736

[4] Wright, H. S., Oh, D. Y., Westhelle, C. H., Fisher, J. L., Dyke, R. E., Edquist, K. T., Brown, J. L., Justh, H. L., and Munk, M. M., "Mars Aerocapture Systems Study," 2006.

[5] Johrisor, W. R., and Longuski, J. M., “Mars Aerocapture Using Bank Modulation,” 2000.

[6] Lockwood, M. K., “Neptune Aerocapture Systems Analysis,”, No. August, 2004, pp. 1-16. doi:10.2514/6.2004-4951.

[7] Braun, R., Spencer, D., Kallemeyn, P., and Vaughan, R., "Mars Pathfinder Atmospheric Entry Navigation Operations," 22nd Atmospheric Flight Mechanics Conference, Vol. 36, No. 3, 1997. doi:10.2514/6.1997-3663, URL http://arc.aiaa.org/ doi/10.2514/6.1997-3663

[8] Werner, M. S., and Braun, R. D., "Characterization of Guidance Algorithm Performance for Drag Modulation-Based Aerocapture," 40th Annual AAS Guidance, Navigation and Control Conference, 2017, pp. 1-12.

[9] Miller, K. L., Gulick, D., Lewis, J., Trochman, B., and Lyons, D. T., "Trailing Ballute Aerocapture : Concept and Feasibility Assessment,”, No. July, 2003. doi:10.2514/6.2003-4655.

[10] Putnam, Z. R., and Braun, R. D., "Drag-Modulation Flight-Control System Options for Planetary Aerocapture," Journal of Spacecraft and Rockets, Vol. 51, No. 1, 2014, pp. 139-150. doi:10.2514/1.A32589, URL/http://arc.aiaa.org/doi/10. 2514/1.A32589 